\title{
Arbor
}

\section{El Sistema Nacional de Salud de los ciudadanos y de los trabajadores}

\section{Jesús Pérez Martínez}

Arbor CLXXX, 710 (Febrero 2005), 383-388 pp.

La Constitución Española establece para la ciudadanía el derecho a las prestaciones de la Seguridad Social en su artículo 41 y el derecho a la protección de la salud en el artículo 43. Ambos derechos se garantizan mediante el desarrollo de sendos sistemas públicos, el de la Seguridad Social y el Nacional de Salud, que desde sus inicios hasta el momento actual han sido factores principales en la redistribución de la riqueza, la cohesión social y la eficacia productiva y social.

Las prestaciones de asistencia sanitaria, incluidas en la acción protectora de la Seguridad Social y, en su origen, financiadas con cargo a su presupuesto, adquirieron la extensión universal con la Ley General de Sanidad (LGS) en 1986 y la naturaleza no contributiva, a partir de la Ley de Consolidación y Racionalización del Sistema de Seguridad Social en 1997. A partir de este momento, las prestaciones y servicios de asistencia sanitaria se financiaron por el Estado, hasta que en 2001 la financiación sanitaria se integra en la financiación general de las Comunidades Autónomas.

El Sistema Nacional de Salud (SNS), definido y creado en la LGS, como el conjunto de los Servicios de Salud de la Administración del Estado y de los Servicios de Salud de las Comunidades Autónomas, ha experimentado, a lo largo de su historia, cambios en cuanto a su financiación, prestaciones y niveles asistenciales, acompañados de modificaciones en la gestión, en el marco normativo y en el modelo de participación social. Se ha constituido como un modelo público, con cobertura universal, equitativo y solidario, en el que el acceso al sistema y a las prestaciones sanitarias deben realizarse en condiciones de igualdad efectiva -equidad-, 
y la política de salud debe estar orientada a la superación de los desequilibrios territoriales y sociales. Paralelamente, desde el año 1981 hasta 2001, la gestión de la asistencia sanitaria se fue descentralizando hasta culminar las transferencias en esta materia coincidiendo con el nuevo modelo de financiación de la sanidad.

Sin embargo, el análisis del sistema sanitario nos permite afirmar que no siempre se cumplen estas condiciones de universalidad, equidad y solidaridad, y se pueden destacar algunos aspectos mejorables que deben subsanarse: como que todavía queda sin derecho a la asistencia sanitaria un porcentaje muy reducido de la población, que persisten subsistemas no integrados de provisión privada de servicios y que se incrementan las desigualdades interterritoriales, en un contexto en el que la financiación se manifiesta como claramente insuficiente. Sin embargo, ninguno de estos aspectos sería preocupante, si existieran mecanismos eficaces correctores de las desigualdades orientados hacia la equidad y garantes de un reparto presupuestario, según las necesidades, y si el sistema de financiación sanitaria, en el que hoy los recursos no tienen carácter finalista, fuera el adecuado.

La UGT ha insistido, desde septiembre de 2001, en relación con al Acuerdo de Financiación Autonómica y Sanitaria del Consejo de Política Fiscal y Financiera y el modelo de financiación autonómica y sanitaria, con una serie de consideraciones y propuestas para contribuir al sostenimiento del SNS y de las prestaciones sanitarias de la Seguridad Social. A lo largo de los últimos tres años, este sistema de financiación autonómico no se está demostrando como el más apropiado para garantizar la sostenibilidad del sistema sanitario público y, a nuestro juicio, para determinar un mejor diseño del mismo, se debe partir de una concepción común del sistema sanitario, calcular los recursos y las fuentes necesarios para su financiación. Sin olvidar que la sostenibilidad del sistema también depende de una mejora de su gestión pública en todos los ámbitos.

Por otra parte, con respecto al gasto sanitario público se observa que crece cada año y lo hace con el aumento de la población y de la edad, con las necesidades de tecnología, de personal, y de consumo farmacéutico, principalmente, poniendo de manifiesto la insuficiencia financiera de un sistema de financiación que nació junto con las diez últimas transferencias sanitarias a las Comunidades Autónomas, efectuadas en bloque en una única decisión política. Sin embargo, en España el gasto sanitario público por persona y año es uno de los más bajos de la Unión Europea de $\operatorname{los} 15$, lo que pone de manifiesto que no se trata sólo ni primordialmente de un problema de financiación, sino de adoptar decisiones políti- 
cas para defender uno de los pilares fundamentales del Estado de Bienestar.

La UGT cree firmemente que el derecho a la salud que tienen todos los ciudadanos sólo puede hacerse efectivo, mediante un Sistema Nacional de Salud con financiación, provisión y gestión públicas que, como tal, garantice, por un lado, la aplicación de los principios básicos de universalidad, equidad, gratuidad y eficacia y, por otro, tenga al usuario como centro del mismo. Unas garantías igualmente vivas con la descentralización sanitaria ${ }^{1}$.

Además, en el sistema sanitario público se detectan tensiones que no son achacables únicamente a las deficiencias de financiación, sino que son consecuencia de una gestión plagada de dificultades que se agravan con el dilatado proceso de transferencias, dando lugar a situaciones territoriales bien diferentes que ponen en peligro la equidad del sistema y manifiestan desigualdades. La descripción de los diferentes servicios autonómicos de salud nos proporciona un mapa en el que se incrementan cada vez más las diferencias interterritoriales para el acceso a las prestaciones, las condiciones de salud, el gasto "per cápita» en salud por habitante, los recursos tecnológicos y la dotación de personal.

En el largo proceso de descentralización y transferencias se han puesto de manifiesto problemas en la distribución competencial entre la Administración General del Estado y las Comunidades Autónomas, así como de coordinación en el SNS. También se observan claros síntomas de desestructuración y desmembramiento relacionados con los diferentes modelos de gestión, con diferencias en las condiciones de trabajo y signos de desmotivación generalizada en el personal, que permanecerán sin solución mientras no se determinen los mecanismos de gobierno, coordinación y cohesión necesarios para el mantenimiento del sistema.

Para que los ciudadanos puedan acceder a las prestaciones sanitarias en condiciones de igualdad, con independencia de su residencia y movilidad en todo el territorio, es fundamental que los servicios sanitarios dispongan de los recursos necesarios, materiales y humanos, y que funcionen como un sistema, integrado y coordinado. Pero, además, las prestaciones sanitarias de la Seguridad Social contempladas en la Ley de Cohesión y Calidad del SNS (LCCSNS), deben ser objeto de un desarrollo reglamentario que establezca el catálogo y la cartera de servicios común en el conjunto del sistema.

Para la UGT la incorporación que la LCCSNS hace de la Salud Pública como prestación ha sido un avance. En la actualidad la Salud Pública está transferida a las CCAA donde todavía no está suficientemente desarrollada ni integrada con los servicios asistenciales de Atención Pri- 
maria y Especializada, perdiéndose la potencialidad que ofrece como instrumento de planificación estratégica de la sanidad, de utilización adecuada de los recursos, de promoción, de prevención y de restauración de la salud.

Para ajustar el modelo a los principios que lo inspiran, la UGT constata la necesidad de realizar un conjunto de tareas pendientes, como la consolidación de una Atención Primaria, que no se reduzca a la atención de la enfermedad, la reducción drástica de las listas de espera, la inmediatez de las pruebas diagnósticas y un potente sistema de desarrollo de las políticas de salud pública y laboral ${ }^{2}$.

En relación con la prestación farmacéutica, es importante analizar y valorar el crecimiento del gasto público en este capítulo y la gestión que se ha realizado en los últimos años en el Ministerio de Sanidad y Consumo y en las CCAA. A la vista de los resultados, es fácil concluir con la necesidad de establecer medidas para el uso racional del medicamento y la aplicación de medidas que mejoren la calidad en la gestión de la prestación en su conjunto (autorización de medicamentos, distribución, prescripción, seguimiento del tratamiento, innovación terapéutica, etc.) y evaluar sus efectos, como la contención del gasto farmacéutico, entre otros.

En la Unión Europea se disfruta de niveles altos de salud y de bienestar social fruto de las políticas de protección social que han ido desarrollando los sistemas sanitarios públicos. Estos sistemas, con origen y características diferentes en cada país, se han reformado con el objetivo de mejorar la calidad de sus servicios y hacer frente a las nuevas necesidades, pero todavía están lejos de la convergencia en materia de salud y bienestar. En este ámbito, las políticas en materia de Salud Pública y consumo son las protagonistas, no existiendo iniciativas políticas en relación con los sistemas sanitarios de los diferentes países, salvo en lo relacionado con la movilidad de los pacientes y profesionales sanitarios y las actividades transfronterizas. En cuanto a las relaciones entre los diferentes sistemas sanitarios, es fundamental el papel que desempeña la Seguridad Social en relación con la libre circulación de los ciudadanos y la atención sanitaria en la UE, principalmente con la tarjeta sanitaria para la identificación personal y el acceso a la asistencia sanitaria y la facturación entre sistemas.

En este contexto europeo, comparativamente, nuestro SNS es eficiente, porque con una financiación menor que en la mayoría de los países del entorno y con unos recursos humanos que empiezan a escasear, insuficientes para la demanda actual, se obtienen buenos resultados. 
En la «Declaración de Toledo sobre el Derecho a la Salud para todos en la Unión Europea», en Diciembre de 2003, se afirma que «los criterios de convergencia económica están determinando el desarrollo y sostenimiento de los actuales niveles del Estado de Bienestar. Las medidas de contención del gasto público tienen un impacto negativo sobre el gasto social. No sólo importa el nivel global del gasto público, sino también su distribución». La UGT, como firmante de esa declaración y defensora de todo su contenido, es plenamente consciente de ello y defiende que se establezcan criterios e instrumentos de cooperación para garantizar el derecho a la salud, fortalecer la cohesión y lograr la convergencia europea en materia de políticas públicas de bienestar social y sanitaria.

Nuestro sistema sanitario ha sido y es el refugio de muchos ciudadanos en situación de dependencia, sobre todo para los mayores, con necesidades sociosanitarias que van mas allá de las propias del sistema sanitario. En este sentido, es imprescindible el desarrollo de la prestación sociosanitaria con servicios suficientes para la convalecencia, para los enfermos crónicos y para la salud mental, especialmente mediante el desarrollo de la enfermería y otros cuidados a domicilio, con continuidad y proximidad al entorno socio-familiar del paciente y de la creación de un Sistema Nacional de Dependencia, complementario al Sistema Nacional de Salud, para el que se establezca un catálogo de prestaciones y servicios públicos que puedan contribuir a la satisfacción de la demanda sobre algunos niveles asistenciales, imposibles de satisfacer con medios privados para la inmensa mayoría de la población.

Por último, unas cuantas consideraciones en cuanto a la participación institucional de los interlocutores sociales (Organizaciones Empresariales y Sindicales) más representativos, según la denominación de la Ley Orgánica de Libertad Sindical (LOSL) que está dispersa, en órganos de muy diferente composición y funciones y sobre la que hay que plantearse su reordenación, con el objetivo de recuperar el tripartismo y la paridad en los órganos de información, consulta y participación en las Administraciones públicas, según los criterios de la OIT y de la mencionada LOSL.

Nuestro papel en los órganos de participación institucional, como representantes de los trabajadores, como Sindicato, es un pilar fundamental del Estado de Bienestar que, arrancando y siguiendo anclado a la S.S., se extiende y se hace universal, consiste en la responsabilidad de que también representamos a todos los ciudadanos, además de que representemos a los trabajadores, que son la inmensa mayoría de los ciudadanos.

A modo de conclusiones generales, para reforzar el SNS como un ristema sanitario público, gratuito, universal, equitativo y solidario de- 
ben establecerse mecanismos políticos y adoptarse medidas eficaces, como los siguientes:

- Equiparar el gasto social en España a los parámetros de la UE15, como condición necesaria del progreso económico y social y, consecuentemente, incrementar al menos en un punto en porcentaje sobre PIB del gasto sanitario público.

- La descentralización del sistema sanitario debe ser compatible con la vertebración y coordinación que se precisa para asegurar la equidad y la solidaridad interterritorial, para lo que es necesaria una mejora de los sistemas de información y de los sistemas para compensar los desequilibrios territoriales y sociales. Por ello, estimamos que el Fondo de Cohesión debería convertirse en un Fondo de la Seguridad Social para la Cohesión del SNS, ampliando su dotación, cambiando su naturaleza e incluyendo entre sus fines, de manera prioritaria, la corrección de desigualdades.

- En este contexto, debería desarrollarse el Plan Integrado de Salud del SNS para marcar los objetivos y orientar las actuaciones del conjunto del Sistema y las asignaciones del Fondo de Cohesión.

- Por tratarse de un pilar fundamental del Estado de Bienestar, debe potenciarse la participación social a todos los niveles y mejorar la calidad de nuestra representación institucional, según el modelo establecido en la Ley de Cohesión y Calidad del SNS y originado en la Seguridad Social que posibilita la participación y representación de la ciudadanía.

Por último, la UGT ha propuesto la apertura de un capítulo específico sobre el sistema sanitario en el Diálogo Social, en el marco de la Declaración del 8 de julio, en paralelo con el Diálogo comprometido por el Gobierno sobre capítulos del Estado de Bienestar tan transcendentes como el sistema de pensiones y de prestaciones de la Seguridad Social, y un sistema de atención a la dependencia. Diálogo Social para situar la cohesión social del SNS en el corazón de la prestación sanitaria, más allá de la necesidad de acordar nuevos sistemas de financiación suficiente para los Servicios Públicos de Salud que gestionan las Comunidades Autónomas.

\section{Notas}

1 Programa Fundamental. 38 Congreso Confederal. Marzo de 2002.

2 Programa Fundamental. 38 Congreso Confederal. Marzo de 2002. 